\title{
First-light instrument for the 3.6-m Devasthal Optical Telescope: 4Kx4K CCD Imager
}

\author{
Shashi Bhushan Pandey ${ }^{1 *}$, Rama Kant Singh Yadav ${ }^{1}$, Nandish Nanjappa ${ }^{1}$, \\ Shobhit Yadav ${ }^{1}$, Bheemireddy Krishna Reddy ${ }^{1}$, Sanjit Sahu ${ }^{1}$, \\ Ramaiyengar Srinivasan ${ }^{2}$ \\ ${ }^{1}$ ARIES, Manora Peak, Nainital, UK, 263001 \\ ${ }^{2}$ Vemana Institute of Technology, Mahayogi Vemana Road, Bangalore, 560034
}

\begin{abstract}
As a part of in-house instrument developmental activity at ARIES, the $4 \mathrm{~K} \times 4 \mathrm{~K}$ CCD Imager is designed and developed as a first-light instrument for the axial port of the 3.6-m Devasthal Optical Telescope (DOT). The $\mathrm{f} / 9$ beam of the telescope having a plate-scale of $\sim 6.4^{\prime \prime} / \mathrm{mm}$ is utilized to conduct deeper photometry within the central $10^{\prime}$ field of view. The pixel size of the blue-enhanced liquid nitrogen cooled STA4150 $4 \mathrm{~K} \times 4 \mathrm{~K}$ CCD chip is $15 \mu \mathrm{m}$, with options to select gain and speed values to utilize the dynamic range. Using the Imager, it is planned to image the central $\sim 6.5^{\prime} \times 6.5^{\prime}$ field of view of the telescope for various science goals by getting deeper images in several broad-band filters for point sources and objects with low surface brightness. The fully assembled Imager along with automated filter wheels having Bessel $U B V R I$ and SDSS ugriz filters was tested in late 2015 at the axial port of the 3.6-m DOT. This instrument was finally mounted at the axial port of the 3.6-m DOT on 30 March 2016 when the telescope was technically activated jointly by the Prime Ministers of India and Belgium. It is expected to serve as a general purpose multi-band deep imaging instrument for a variety of science goals including studies of cosmic transients, active galaxies, star clusters and optical monitoring of X-ray sources discovered by the newly launched Indian space-mission called ASTROSAT, and follow-up of radio bright objects discovered by the Giant Meterwave Radio Telescope.
\end{abstract}

\section{Introduction}

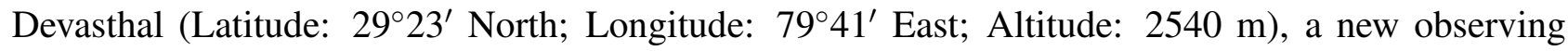
station of the Aryabhatta Research Institute of Observational Sciences (ARIES), is well suited for astronomical observations at optical and near-infrared wavelengths in terms of seeing (10\% of the nights, the FWHM seeing is $\left.0.7^{\prime \prime}\right)$, atmospheric stability ( $\sim 210$ spectroscopic and 160 photometric nights per year), and having excellent logistics for any high altitude observational facility (Pant et al. 1999, Stalin et al. 2001). Research activities in many galactic and extragalactic scientific areas have been conducted at ARIES during last few decades (Pandey \& Mahra 1986, Stalin et al. 2004, Joshi et al. 2006, Yadav et al. 2008) using meter-class telescopes. ARIES Nainital has a longitudinal advantage as it lies in the middle of the $180^{\circ}$ wide belt between the Canary Islands $\left(\sim 20^{\circ} \mathrm{W}\right)$

*shashi@aries.res.in 
and Eastern Australia $\left(\sim 160^{\circ} \mathrm{E}\right)$ for observations of transients and time-critical astronomical events like gamma-ray bursts (GRBs), supernovae (SNe), and micro-lensing events (Sagar \& Pandey 2012, Pandey 2006, Joshi et al. 2005).

The recently installed DOT has a f/2 primary mirror M1 with a diameter of $3.6 \mathrm{~m}$, a secondary mirror M2 with a diameter of $0.9 \mathrm{~m}$ and an effective focal ratio of $\mathrm{f} / 9$, a Ritchey-Chretien configuration, and an alt-azimuth mounting. It has a field of view of $10^{\prime}$ without corrector at the axial port, and an unvignetted field of $30^{\prime}$ for the axial port and of $10^{\prime}$ for the two side ports. The tracking accuracy of the 3.6-m DOT is smaller than $0.1^{\prime \prime}$ root-mean-square (RMS) for less than one minute in open loop and smaller than 0.1" RMS for about one hour in closed loop mode (see Sagar et al. 2012 and other articles in these proceedings for more details). A cylindrical space of minimum 2.5 meter below the focal plane for axial port and approximately 3.0 meter diameter around optical axis will be available for the instrument envelope. Several first-generation back-end instruments were proposed for the 3.6$\mathrm{m}$ DOT for broad-band imaging and spectroscopy covering the 350-3600 $\mathrm{nm}$ wavelength range, i.e. (1) $4 \mathrm{~K} \times 4 \mathrm{~K}$ CCD optical general purpose Imager for deeper photometric observations, (2) TIFR nearinfrared imaging camera (TIRCAM2), (3) ARIES Devasthal Faint Object Spectrograph and Camera (ADFOSC), (4) high-resolution Echelle spectrograph, (5) TIFR-ARIES near-infrared spectrometer (TANSPEC), and (6) multi-integral field unit optical spectrograph (DOTIFS).

In this article, we give an overview of the design and development of the first-light instrument called $4 \mathrm{~K} \times 4 \mathrm{~K}$ CCD optical Imager along with some of the proposed science goals. Also, we briefly describe the results of the characterization of the instrument and some of the preliminary science results based on the multi-band imaging performed with the CCD Imager mounted at the axial port of the 3.6-m DOT.

\section{Science Objectives}

Deeper imaging of galactic and extragalactic point sources and objects with low surface brightness could be performed using the $4 \mathrm{~K} \times 4 \mathrm{~K}$ CCD Imager in several broad-band filters (set of Bessel $U B V R I$ and SDSS ugriz filters) at the axial port of the 3.6-m telescope. Broad areas of various science objectives are briefly described in the following sub-sections.

\subsection{Star clusters as a tool for stellar evolution}

A group of gravitationally bound stars formed nearly at the same time from the molecular cloud are known as star clusters. They are therefore located at the same distance and also considered to have same primordial chemical composition. Consequently, the Hertzsprung-Russell (HR) diagram of a star cluster reflects the evolutionary dispersion of approximately equally old stars of different masses, after a lapse of time specified by the age of the cluster. Open clusters and globular clusters therefore provide a wealth of information against which to test the theory of stellar evolution. Comparison between the theoretical and observed HR diagrams leads to the determination of the age and chemical composition of stars in the cluster (Sagar et al. 1986, Friel 1995, Yadav et al. 2008, Saurabh et al. 2012). By using multi-band data, it has been observed that most of the metal rich globular clusters in our galaxy are concentrated towards the Bulge and are severely affected by interstellar absorption (Sagar et al. 1999, Alonso-Garcia, J. et al. 2012) except in the Baade's window (Stanek 1996). Consequently, only a few have been observed down to the magnitude of the turn-off point in the colour magnitude diagram (CMD), and hence their ages are unknown. Use of infrared fluxes along with optical should allow a more precise conversion from observed to theoretical parameters and hence improved isochrone fitting to be made. It should thus be possible to determine the ages of the 
metal rich clusters with high precision and this will allow a comparison to be made with the ages of the metal poor clusters (William \& Rene 1979, Bekki \& Forbes 2006, Kalirai et al. 2008).

\subsection{PMS stars and star formation in star clusters}

The presence of dust and gas within young star clusters and in their immediate vicinity is well established. Therefore their properties and the role in the evolution of a cluster are crucial in understanding star formation as well as cluster formation mechanism. The knowledge of extinction properties in these systems is necessary to determine accurate physical parameters using optical data along with data taken at near-infrared wavelengths. Young open clusters (YSCs; age $<10 \mathrm{Myr}$ ) still embedded in the parent nebulous regions, which are neither evolved into stellar remnants nor escaped the gravitational grasp of the molecular cloud and/or dense stellar cluster, present an unique laboratory for understanding the process of star formation, its history, the early evolution of stars over a wide mass range, and the nature of interaction of young stars with their surrounding interstellar medium. These regions contain stars of high mass as well as low mass pre-main sequence (PMS) stars and the characteristics of their physical properties along with the distribution of ionized gas and molecular cloud under the influence of high mass stars can give us clues about the physical processes that govern their formation. The broad mass range of the cluster members can also be used to quantify the relative numbers of stars in different mass bins by using optical HR diagrams and to find systematic variations of the initial mass function (IMF) with different star-forming conditions. Identifying systematic variations of star formation would allow us to understand the physics involved in assembling each of the mass ranges and thus to probe early cosmological events (Kroupa 2002). IMFs of young clusters in the mass range of about $\sim 30 \quad 0.6 \mathrm{M}_{\odot}$ can be represented by a power law with a break around $1-2 \mathrm{M}_{\odot}$. It is found that the IMF varies from one cluster region to another (Saurabh 2012). The evolutionary sequences and the nature of these PMS stars are still not understood. The imaging of such objects would be able to determine their age correctly and will also be helpful to constrain the mass-function towards the lower mass side (Pandey et al. 1997, Pandey et al. 2008).

\subsection{Study of GRB afterglows and Supernovae}

Recent studies suggest that the observed GRBs and/or core-collapse supernovae (CCSNe) occur due to the conversion of the kinetic energy in relativistic out-flowing ejection from the progenitor (i.e. central engine of the GRB or so called fireball) to non-thermal gamma-ray/X-ray radiation via internal shocks. The subsequent interaction of the ejected material with the surrounding medium through external shocks produce afterglows visible in all bands from X-rays to radio wavelengths which could be predominantly thermal radiation in case of CCSNe. Afterglow observations of GRBs/CCSNe are of crucial importance in understanding this highly energetic, cosmological, ultra-relativistic phenomenon. Observations of optical afterglows play a unique role in understanding the nature of their progenitor, environment, and energetics (Pandey et al. 2003, Sagar \& Pandey 2012). Usually the afterglow of GRBs are observed at $\sim 16-18 \mathrm{mag}$ and fainter (Kann et al. 2011). In few cases, afterglows are very faint (i.e. $23 \mathrm{mag}$ and fainter). Photometric measurements are also necessary to know more about the surroundings of the progenitors, nature of the dark GRBs and the host galaxies (Kumar \& Zhang 2015). The correlation of some of the GRBs with SNe could also be probed using photometric observations at late times i.e. $\sim 15(1+z)$ days, where $z$ is the measured red-shift of the burst. Optical afterglows of short-duration GRBs, Kilonovae and other interesting energetic transients and newly discovered various types of Supernovae are also aimed to be monitored with the $4 \mathrm{~K} \times 4 \mathrm{~K}$ CCD Imager at the 3.6-m DOT (Pandey 2013). 


\subsection{Galaxies and Optical variability of powerful active galactic nuclei}

Much progress in extragalactic research and cosmology rests on a detailed knowledge of the properties of nearby galaxies. Deep CCD photometry gives direct evidence of various star-forming regions and dust lanes in the galaxies and also incidence of merger or interaction of galaxies. There is still a large sample of galaxies to be studied. Wolf-Rayet galaxies are another important class of objects, both in terms of understanding massive star evolution and star burst phenomenon. Also, optical intensity fluctuations of radio-loud quasars on an hour-like time scale is now a well established phenomenon. In contrast, it is not at all clear if similar intra-night variations are also exhibited by radio-quiet quasars which comprise nearly $80 \%$ of the quasar population. Such observations can provide valuable clues to the question of radio dichotomy of quasars, currently among the most outstanding issues in extragalactic astrophysics. The significance of intra-night variability studies lies in the fact that they enable a probe of the innermost nuclear cores of active galaxies, on the scale of mas (micro-arcseconds) which are totally beyond the reach of any imaging techniques currently in use. A multi-wavelength study of rapid intensity variations of active galactic nuclei (AGNs) thus provides a uniquely powerful tool for investigating the process occurring in the vicinity of their central engines. Given the widespread perception that most radio-quiet objects lack relativistic jets, the likely sources of these small fluctuations are the accretion disks that almost certainly exist around central super massive black holes. However, no consensus has been reached about how similar the intra-night optical variability characteristics are for radio-quiet and radio-loud quasars (Stalin et al. 2004, Fabian 2012).

\subsection{Studies of EUV-bright and soft X-ray sources}

So far only about $35 \%$ of the sources of the X-ray satellite ROSAT have been identified with known optical objects. The optical identification content of these sources is equally deficient (Pasquini \& Belloni 1991). Extrapolating from the number and types of sources that have been identified so far, it is expected that about $50 \%$ of these sources will be AGNs or quasars, $35 \%$ will be stars, $10 \%$ will be normal galaxies and clusters of galaxies, and about $5 \%$ other objects. The error circle associated with the position of an X-ray source depends on the instrument used, the characteristics of the field, and the strength of the source. Positional information available from central regions of the field of view of imaging X-ray telescopes (e.g. ROSAT) usually has errors ranging from about $5^{\prime \prime}$ to $20^{\prime \prime}$ depending on the type of detector used at the focus of the telescope. With error circles of $20^{\prime \prime}$, there is usually no more than one object brighter than 19.5 mag (Pandey et al. 2005) in the $V$-band within the error circle, thus targets can be identified very easily using images taken with a CCD on a 3.6-m telescope and taking short exposures of tens of seconds to a minute. Many new soft Xray sources have recently been discovered in surveys with the Einstein and ROSAT space missions. A large fraction of these objects are probable new accretion binaries, either magnetic cataclysmic variables or low mass X-ray binaries. Our aim will be to identify the optical counter parts and then to carry out detailed optical/near-IR photometric and spectroscopic studies. The optical follow-up observations of the ASTROSAT sources would also be a key target for the $4 \mathrm{~K} \times 4 \mathrm{~K}$ CCD Imager (Sreekumar 2005).

\subsection{Optical identifications of radio sources from GMRT}

The Giant Metrewave Radio Telescope (GMRT) has the potential of placing India in a dominant position in the world of low-frequency radio astronomy. Optical observations of some of these radio sources with the 3.6-m DOT is another obvious scientific goal (Samuel \& Ian 2011). Finding the optical/near-infrared counterparts of these radio sources is going to be the first essential step in ascertaining their nature and isolating the most promising objects among them, which could then be taken to 6-m to 10-m class telescopes for a detailed study. Without these optical identifications, the 
science targets of GMRT would remain largely unfulfilled and thus a guaranteed access to medium to large-size optical telescopes is required. The CCD Imager along with the modern 3.6-m DOT could provide sufficiently deep detections ( $\mathrm{B} \sim 25 \mathrm{mag}$ ) within an hour of exposure, thus yielding optical identifications for the majority of the radio sources detected with GMRT (Swarup et al. 1991, Singal et al. 2004, Cameron et al. 2005).

\section{$3 \quad 4 K \times 4 K$ CCD Imager}

The $4 \mathrm{~K} \times 4 \mathrm{~K}$ CCD Imager with motorized filter-housing and camera mounting arrangements is designed to be mounted at the axial port of the 3.6-m DOT. The $\mathrm{f} / 9$ beam of the telescope system is directly used without any focal reducer and has a plate-scale of $\sim 6.4^{\prime \prime} / \mathrm{mm}$. It is planned to use the f/9 beam directly to utilize the central unvignetted $\sim 10 \times 10^{\prime 2}$ of the science field using appropriate filters.

The design of the Imager and its various sub-components were prepared indigenously at ARIES. A suitable blue-enhanced $4 \mathrm{~K} \times 4 \mathrm{~K}$ CCD camera with a pixel size of $15 \mu \mathrm{m}$ and associated Archon control electronics was purchased in 2012 as demanded for the proposed science goals and availability of such CCDs in the market. The required mechanical design, manufacturing and filter wheel motorization were completed by a team of engineers and scientists at ARIES. A schematic block diagram of the CCD Imager is shown in Fig. 1. The details about development of various phases of the $4 \mathrm{~K} \times 4 \mathrm{~K} \mathrm{CCD}$ Imager project are described below.

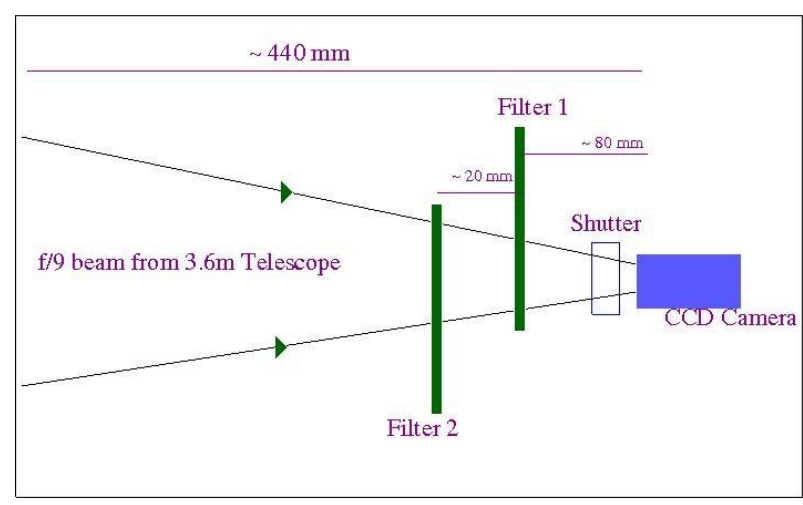

Schematic diagram of the proposed CCD imager (Not to scale)

Figure 1: Schematic diagram of the $4 \mathrm{~K} \times 4 \mathrm{~K}$ CCD Imager.

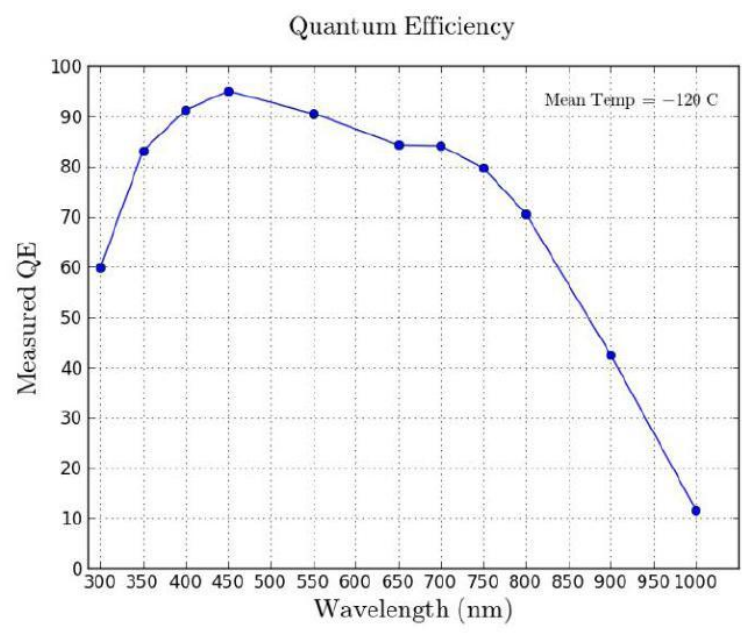

Figure 2: Measured values of the quantum efficiency as a function of wavelength for the STA $4 \mathrm{~K} \times 4 \mathrm{~K}$ CCD chip.

\subsection{Zemax Analysis}

The f/9 beam at the axial port of the 3.6-m DOT was used to analyze the image quality due to insertion of filters, possible tilt of the filters and tilt of CCD chip plane using Zemax software ${ }^{1}$. One of the two filters (fused silica with 5-mm thickness) is inserted in the optical path of the telescope before the focal plane. The focal plane of the telescope is shifted by $1.58 \mathrm{~mm}$ away from the actual focal plane due to the 5-mm filter thickness whereas the change in the image quality is insignificant. This image shift

\footnotetext{
${ }^{1}$ http://http://www.zemax.com//
} 
can be compensated by moving M2 in the z-axis direction. Filters were also tilted by $1^{\circ}$ individually and the image quality is observed. No significant change in the RMS radii is observed. There is a change of around $4 \mu \mathrm{m}$ in RMS radii for a tilt of $5^{\circ}$. It is to be note that the tilting is done in one direction only by assuming that the system is symmetric.

Further, the CCD plane was tilted by around $11^{\prime}$ (equivalent amount of flexure) under the Zemax environment and the image quality was observed. As a result, a change of 4-5 $\mu \mathrm{m}$ in the RMS radii at the extreme/corner field points was noticed which becomes significant if the tilt is increased. The spot diagrams related to the present analysis are described in Figs. 3 and 4.

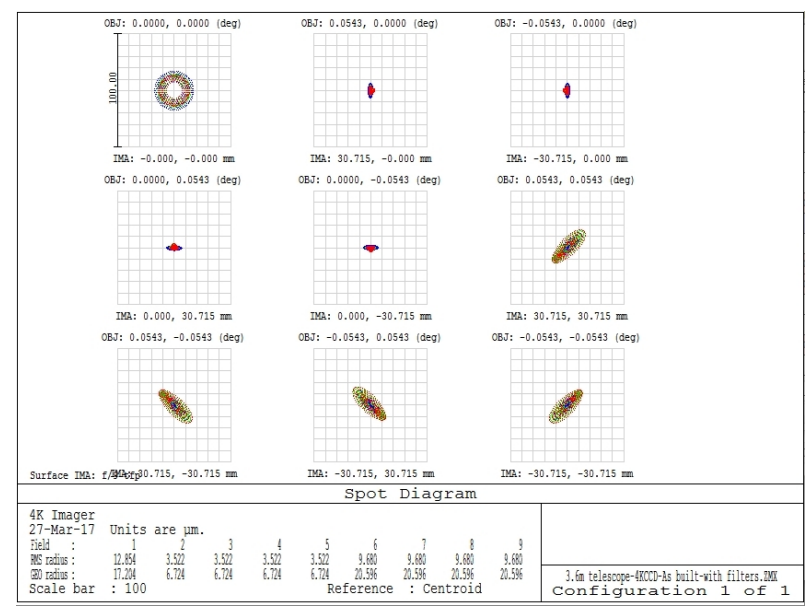

Figure 3: Spot diagram of the 3.6-m DOT as produced by the Zemax software using the f/9 beam at the axial port with a 5-mm thick filter placed in the beam path with the focus compensated by the secondary mirror.

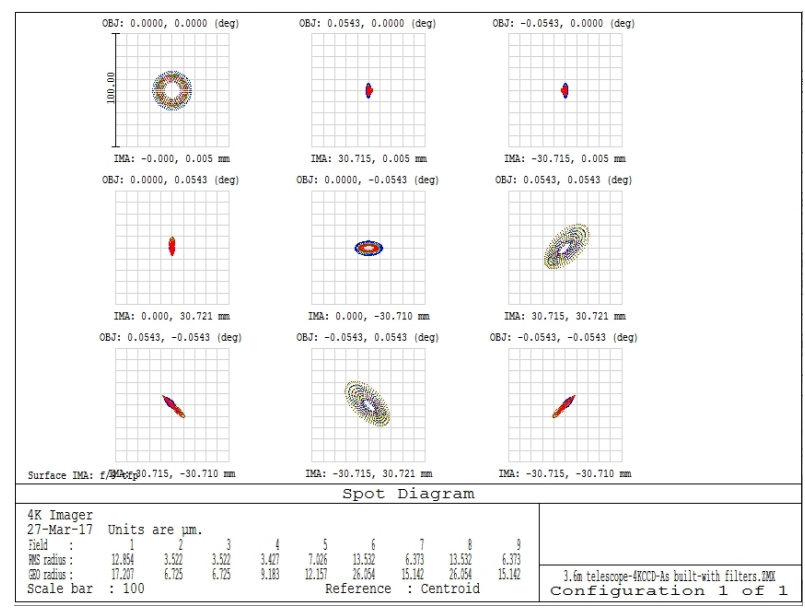

Figure 4: Spot diagram of the 3.6-m DOT as produced by the Zemax software using the $\mathrm{f} / 9$ beam at the axial port with a 5-mm thick filter inserted in the path but the CCD plane tilted by $11^{\prime}$ with respect to the normal to optics axis.

\subsection{The CCD detector}

The requirements of a CCD detector/the pixel sizes are mainly governed by the telescope optics and the seeing conditions of the site. At the time of purchase, pixel size of the large format astronomical grade blue-enhanced single chip CCDs in the market were limited to $15 \mu \mathrm{m}$. At Devasthal, the median seeing is $\sim 1.1$ to $1.2^{\prime \prime}$ and it was proposed to use the f/9 beam directly at the axial port of the 3.6-m DOT. So, based on the scientific goals mentioned above, it was decided to purchase a blue-enhanced, back-illuminated $4 \mathrm{~K} \times 4 \mathrm{~K}$ CCD chip with a pixel size of $15 \mu \mathrm{m}$ in 2011 from Semiconductor Technology Associates Inc. (STA) USA. The technical parameters of the STA CCD chip/camera are listed in Table 1 (for more details about the camera, we refer to website of STA ${ }^{2}$ ). The quantum efficiency curve as a function of wavelength is for the STA CCD chip shown in Fig. 2.

For the given plate scale of $6.4^{\prime \prime} / \mathrm{mm}$, a $15-\mu \mathrm{m} 4 \mathrm{~K} \times 4 \mathrm{~K}$ CCD camera is able to image $\sim 6.5 \times 6.5^{\prime}$ of the sky, though images would be over-sampled. So, there is a provision to bin the pixels in various combinations to optimize with seeing values during observations. Binning the pixels further improves the signal-to-noise and the read out would be faster for a given set of read-out noise and gain values. Other sub-components of the $4 \mathrm{~K} \times 4 \mathrm{~K} \mathrm{CCD}$ camera are described below.

\footnotetext{
${ }^{2}$ http://www.sta-inc.net/
} 
Table 1: Overview of the characteristic properties of the $4 \mathrm{~K} \times 4 \mathrm{~K}$ CCD chip STA $4150 \mathrm{BI}$ (number 155727 W12 D01).

\begin{tabular}{|c|c|}
\hline Specifications/ & Values \\
Parameters & $15 \mu \mathrm{m} /$ pixel, 4096x4096 pixels, back-illuminated, 16-bit A-D \\
CCD Chip & $250 \mathrm{k}-265 \mathrm{k}$ electrons, MPP/non-MPP/CAB modes \\
Full-well capacity & $1,2,3,5,10 \mathrm{e}^{-} / \mathrm{ADU}($ selectable $)$ \\
Gain & $10 \%$ to $90 \%$ of the full well $/ 0.999999$ \\
Read-out Noise (@Speed) & $7-9 \mathrm{e}^{-}(@ 1 \mathrm{MHz})$ or $4-6 \mathrm{e}^{-}(@ 500 \mathrm{KHz})$ or $2-3 \mathrm{e}^{-}(\mathrm{KHz})$ \\
Linearity/CTE & $2 \times 3 \times$ or $4 \times 4$ (selectable $)$ \\
Binning & $0.0005 \mathrm{e}^{-} / \mathrm{s}$ at $-120^{\circ} \mathrm{C}$ \\
Dark Current & Liquid Nitrogen cooled, $-121.3^{\circ} \mathrm{C}$ \\
Dewar/cooling & \\
\hline
\end{tabular}

\subsubsection{The Archon controller and communications}

For the STA CCD, the controller is designed and developed in a customized manner and known as Archon controller ${ }^{3}$. The camera controller software is written in QT Version-5. The Archon Controller is connected to Port $1 \& 2$ of lightguide interconnection unit LIU-2 by a LC-LC multimode patch cord. LIU-2 is connected to LIU-1 through a 12 core optical fiber cable which runs through an azimuth cable wrap. A Hewlett Packard (HP) Gigabit switch is connected to LIU-1 by a LC-LC patch cord and finally the Archon Imager Software PC is connected to HP Gigabit switch by a cat 6 Ethernet cable through its 1 GB per/sec Ethernet port (eno2). An Intel 82572 SFP Gigabit Fiber Optical card is also installed in the PC which can also be used to connect the controller. The Archon controller is compatible only with Gigabit ports and thus it should be kept in mind that a Gigabit Ethernet or Fiber port should be there in the Software PC. The controller, mounted along with the camera, operates at $220 \mathrm{~V}$ and $50 \mathrm{~Hz}$ power supply. The power dissipation of the Archon camera controller is $<50 \mathrm{~W}$ to avoid any thermal effects near the telescope during observations.

\subsubsection{Cooling mechanism}

A liquid nitrogen (LN2) cooling mechanism is used to achieve CCD temperatures as low as $\sim$ $120^{\circ} \mathrm{C}$ to minimize the dark current to possible low values, good for longer exposures detecting fainter objects. Also, the liquid nitrogen dewar along with the camera controller was well-suited to the given specifications of the mounting arrangements. The cooling hold time of the camera dewar is kept of the order of $\sim 15$ hours to get a stable temperature during observations. Before filling LN2, the dewar is evacuated using an Edwards turbo molecular pump below 5 mTorr level. Generally, the dewar takes around 2 hours to reach $-120^{\circ} \mathrm{C}$, the suggested temperature during observations. Separate manuals are provided for further assistance about LN2 filling and possible precautions.

\subsubsection{Shutter}

The $4 \mathrm{~K} \times 4 \mathrm{~K}$ CCD Imager system also needs a shutter mechanism to control the light arriving at the science detector in a given exposure time. One important characteristics of the shutter is its ability to operate uniformly, so that every part of the field obtains the same length of exposure, particularly in case of shorter exposures. In case of the $4 \mathrm{~K} \times 4 \mathrm{~K}$ CCD Imager, it was decided to use $125 \mathrm{~mm}$ Bonn

\footnotetext{
${ }^{3}$ http://www.sta-inc.net/archon/
} 
shutters with a mechanism to run the drivers of the shutter assembly through the CCD controller software ${ }^{4}$.

\subsection{The mechanical assembly of the Imager}

The prime requirement of the opto-mechanical design of the Imager assembly is in particular to mount the optical filters, the CCD camera to hold and move these elements with sufficient precision while the telescope is in motion. The opto-mechanical design of the Imager assembly was simplified as much as possible due to the space limitations, manufacturing complexities, and given weight constraints. The filter wheel assembly of the Imager was mounted at the axial port of the 3.6-m DOT with three spider arms connected to the 2-ton dummy weight structure through a baffle arrangement to prevent stray light. The round discs of the two filter wheels with six slots to mount the set of filters are positioned along with the motors, gears, and couplers for an easy accessibility for maintenance, cleaning, and changing of filters periodically. In the design of the optical mountings, it is important that the clamping forces that constrain the position of the optical filters should not be too large. Mounting stress on the optics can cause surface deformations or birefringence in the optical material, and hence degrade the image quality performance. To avoid these possible issues, all the filters are supported by POM (polyoxymethylene) material. While preparing the mechanical design, care was taken to minimize the effects like differential thermal expansion of the optical and mechanical parts to reduce the possible mounting stress. Finite-element analyses (FEA) were performed to verify the stiffness deformation of the filter wheel assembly due to gravity or the mounting stress on optics. A free version of the analysis software package called "Ansys" was used for the FEA analysis and related calculations.

The mounting arrangements for this instrument were designed considering the available space at the axial port of the Cassegrain focus of the 3.6-m DOT. Mechanical specifications of the 3.6-m DOT dictates that the maximum allowable instrument weight at the cassegrain axial port must be below $2000 \mathrm{~kg}$ with a center of gravity located $800 \mathrm{~mm}$ below the instrument flange. Fig. 5 shows the full mechanical structure of the of the $4 \mathrm{~K} \times 4 \mathrm{~K}$ CCD Imager mounted at the instrument flange. More details about various sub-components of the Imager are given in the following subsections.

\subsubsection{Angle Brackets}

Angle brackets connect the housing structure to the telescope axial dummy weight. The three brackets are very stiff components placed at an angle of $120^{\circ}$ The over all load distribution and stiffness of the instrument depends on the three angle brackets. These angle brackets are mounted at $120^{\circ}$ each, connected with the dummy weight and the filter wheel housing at both ends. They are made of an Aluminum T6 6061 alloy.

\subsubsection{Filter Housing}

The requirement of the filter housing structure is to house and support the components like filter wheel assembly, drive mechanisms, CCD camera and control electronics etc. The filter housing structure should be rigid and stiff enough to take overall weight and other forces acting on it during observations and in idle conditions. Apart from supporting the sub-components, the housing structure has a provision to access the filters for maintenance and cleaning of filters periodically. Two identical hollow bearing shafts of different lengths help to maintain the gap between the filter wheels and to support the filter wheels. The bearing shaft is made of hardened steel and ground to tolerance value of $\mathrm{j} 6$ for bearing fittings. The size of the bearing shafts is $70 \mathrm{~mm}$ and they weigh $\sim 1 \mathrm{~kg}$ each. Specialized

\footnotetext{
${ }^{4}$ http://www.bonn-shutter.de/shutter_types.php
} 
deep groove ball bearing are used for filter wheel drive for a smooth and jerk free rotation of the two filter wheels. The external and internal diameters of the deep groove ball bearings are $100 \mathrm{~mm}$ and $70 \mathrm{~mm}$, respectively, with a breadth of $16 \mathrm{~mm}$.

The housing is combination of machined plates made up of an Aluminum T6 6061 alloy material. The full size of the housing is approximately $833 \times 830 \times 117 \mathrm{~mm}^{3}$. The sole purpose of the design and building plate box structure is to ease the alignment related issues and to address maintenance related requirements. The housing has unique plates at diagonal locations to support and hold the stepper driving mechanisms. Top and bottom plates are used to simply support the filter and CCD camera assembly.

\subsubsection{Filter wheel assembly}

The prime requirements of the filter wheel are to accommodate 10 optical filters and to rotate the position of these filters as desired by observers at a time of observation. The filter wheel is one of the high precise components of this instrument due to requirement of accurate positioning and supporting of filters with low mounting stress. To achieve these requirements, the filter wheels are built by reducing weights as much as possible and precisely cutting the rectangular pockets/slots to accommodate filters. The outer periphery of the filter wheels are cut by module- 1 teeth in precise gear hobbing machine and bearing hole machined at center. To avoid inertial play and stiffness problems materials were scooped while manufacturing the filter wheels. Each filter wheel has 6 slots of size $90 \times 90 \mathrm{~mm}$ with a clear aperture of $85 \times 85 \mathrm{~mm}$. One slot in each filter wheel will be empty to be able to use only one filter at the time of observation. The diameter of each filter wheel assembly is $434 \mathrm{~mm}$ mounted along with SKF bearings and bearing covers for mounting purposes.

\subsubsection{Filter wheel Drive system}

Each filter wheel accommodates five filters at a time and each filter is located at a $60^{\circ}$ index angle. As required during observations, each filter is rotated and positioned several times by maintaining precise tolerance limits. The rotation of filter wheels is controlled by control electronics through a pinion gear, worm gear box (ratio 30, anti-backless Moffet), and electronics sensors attached between the filter wheel and stepper motor drive. The total gear reduction ratio between motor to wheel drive is 540, reducing the error between motor to filters approximately by a factor of 540. The required repeatability accuracy of each filter wheel (a few tens of $\mu \mathrm{m}$ ) is achieved through an anti-backlash gear fitted between filter wheel and the gear box. The muffet gear box, used in the Imager, is a less weight compact precise anti-backlash worm gear system manufactured by Muffet Inc. (UK). The pinion gear used is a module-1 teeth spur gear made of high quality nylon material. The purpose of using nylon material is avoiding residual stress and wear between the teeth of the Aluminum filter wheels and the 24 teeth of the pinion teeth. The stepper motor was used to rotate the filter wheels with the desired precision. The motion of the motors were controlled through a micro-controller based card and were interfaced with a GUI based s/w provided to control the CCD electronics.

\subsubsection{Filters}

There is a provision to use both SDSS ugriz and Bessel $U B V R I$ broad-band filters (in the spectral range 360-1000 $\mathrm{nm}$ ) for imaging purposes along with the $4 \mathrm{~K} \times 4 \mathrm{~K}$ CCD Imager. As per the design of the Imager and with the given space at the mounting flange, the f/9 beam of the telescope required filter sizes of $\sim 90 \mathrm{~mm}$ each. The central wavelengths and band-widths of each of the 10 filters in use are tabulated in column 2 of Table 2 . The transmission coefficients of the all 10 filters were verified in the lab and were found to meet the specifications as tabulated in column 3 of Table 2. 


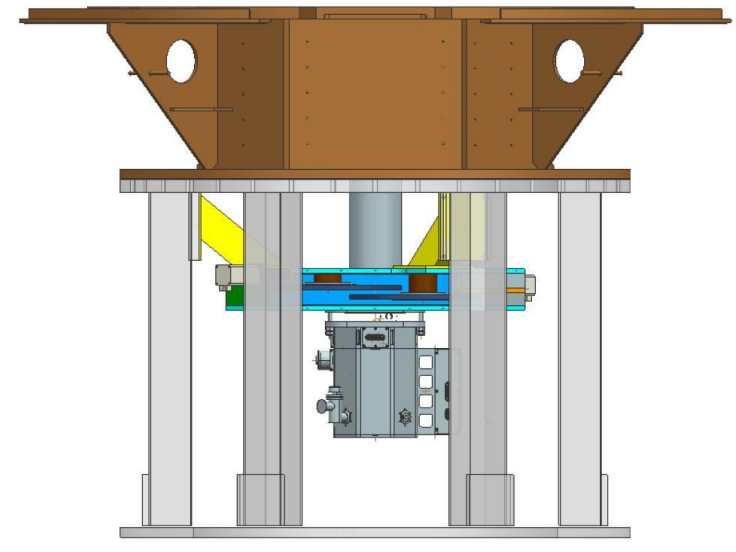

Figure 5: Schematic diagram of the proposed CCD Imager within the given dummy weight and the instrument envelop. The full assembly, including angle brackets, the filter wheel case, motors/gears, and the $4 \mathrm{~K} \times 4 \mathrm{~K} C \mathrm{CD}$ camera are shown in the figure as produced by the software called "pro-e".

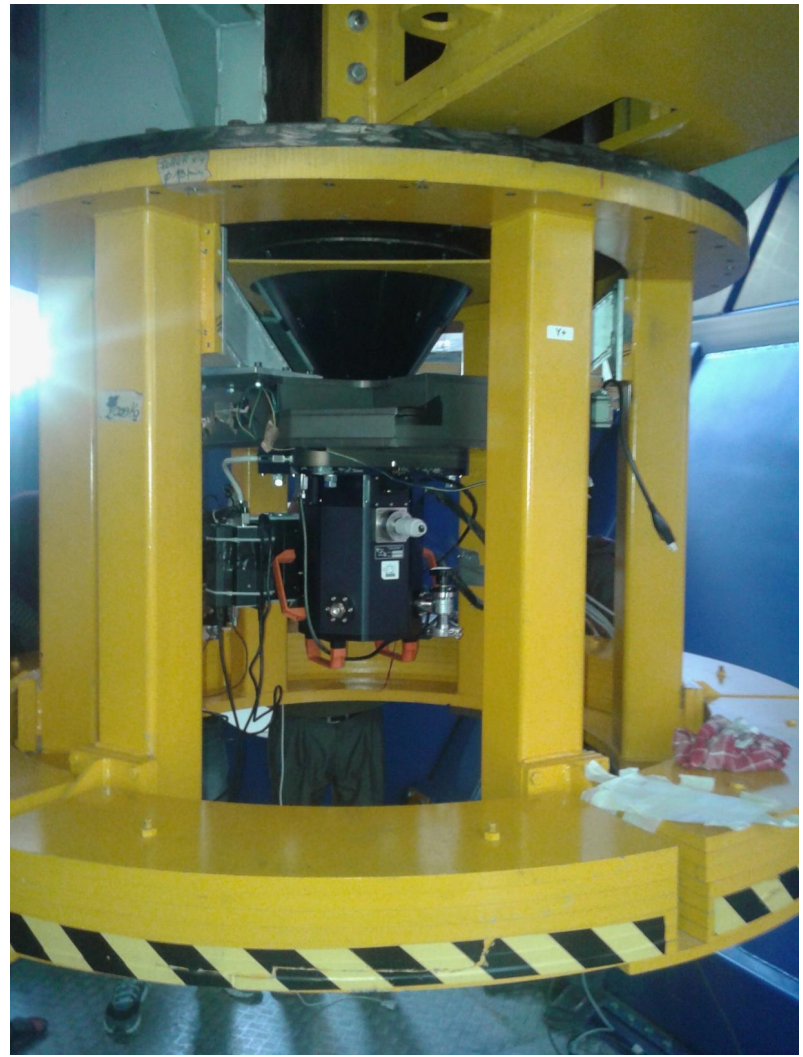

Figure 6: The fully assembled $4 \mathrm{~K} \times 4 \mathrm{~K} \mathrm{CCD}$ camera along with the shutter and automated filter wheels mounted at the axial port of the 3.6-m DOT on 9 December 2015.

\section{Motorized Filter wheel}

The implementation of motorized filter wheels is one of the major in-house activities of the CCD Imager project. The mechanisms (both software and hardware) were designed, developed and implemented solely by ARIES staff members involved in this project. A brief description about this automation is outlined below.

The 3.6-m DOT Imager consists of two filter wheels. Both filter wheels have a separate set of six filter positions namely $U, B, V, R, I, C$ (Clear) and $u, g, r, i, z, c$ (Clear), respectively. A microcontroller based control unit and GUI software are used for the positioning of two filter wheels in this instrument. The control unit consists of a PIC microcontroller having a Serial Communications Interface (SCI) module USART (Universal Synchronous Asynchronous Receiver Transmitter) and a circuit that converts from RS-232 compatible signal levels to the USART's logic levels and viceversa. The control unit communicates with the interfacing PC over TCP/IP protocol using simple ASCII commands through RS-232 to Ethernet converter and vice versa. For moving the wheels stepper motors along with suitable drivers, amplifiers have been employed. Homing is achieved after powering ON using Hall Effect sensors. A detailed log of commands, status, and errors are continuously generated by the GUI software. Both the control unit and the software have been successfully tested and integrated with the Imager instrument.

Initially at the POWER ON, homing is achieved automatically and both filter wheels are positioned at their clear position. Whenever any command is received by the controller, a request of command is executed first. The controller will be ready for the execution of the next command only 
Table 2: Specifications of the 10 broad-band filters (column 1) including the central wavelengths and band-widths (column 2), the transmission percentages (column 3) and the measured values of the atmospheric extinction coefficients using the combination of the CCD Imager and the 3.6-m DOT (column 4).

\begin{tabular}{|l|l|l|l|}
\hline Filters & $\begin{array}{l}\text { Central wavelength/ } \\
\text { Band-width }(\AA)\end{array}$ & $\begin{array}{l}\text { \% Transmission } \\
\text { (measured values) }\end{array}$ & $\begin{array}{l}\text { Extinction coefficients } \\
\text { (measured values in mag) }\end{array}$ \\
\hline Bessel $U$ & $3663 / 650$ & 62.0 & $0.64 \pm 0.03$ \\
Bessel $B$ & $4361 / 890$ & 70.0 & $0.39 \pm 0.02$ \\
Bessel $V$ & $5448 / 840$ & 80.0 & $0.29 \pm 0.02$ \\
Bessel $R$ & $6407 / 1580$ & 88.0 & $0.22 \pm 0.01$ \\
Bessel $I$ & $7980 / 1540$ & 80.0 & $0.17 \pm 0.02$ \\
SDSS $u$ & $3596 / 570$ & 63.0 & $0.70 \pm 0.02$ \\
SDSS $g$ & $4639 / 1280$ & 88.0 & $0.30 \pm 0.04$ \\
SDSS $r$ & $6122 / 1150$ & 85.0 & $0.20 \pm 0.02$ \\
SDSS $i$ & $7439 / 1230$ & 87.0 & $0.14 \pm 0.02$ \\
SDSS $z$ & $8896 / 1070$ & 88.0 & $0.09 \pm 0.03$ \\
\hline
\end{tabular}

after the execution of the previous command. Any request received from the interface PC will be acknowledged after the execution of the request by the control unit. Once the GUI of the filter wheels is enabled and the "CONNECT" button is triggered, the GUI sends a "POWER ON" status request to the control unit. The communication status between interface PC and control unit is displayed depending on the acknowledgment status. Once the communication link is established with the control unit and "POWER ON" status is acknowledged, the GUI sends a current position status request to the control unit. After acknowledgment from the control unit, the current positions of the filter wheels are displayed on the screen. The current position status may be requested from interface PC at any time once the control unit power is energized. The GUI has a provision to request for any individual wheel position movement. If any individual position of the first filter wheel is requested, then before moving the first filter wheel to its requested position, the second filter wheel will be moved to its clear position first if it is not at its clear position and vice versa. During CCD read out time, filter wheels movement may also be requested for the next required filter position.

\section{Throughput Simulations}

The scientific performance of an instrument depends on the maximization of its throughput. The system throughput of the telescope (without a detector like CCD) is expected to be $\sim 60 \%$ for the imaging mode.

The expected counts from a point source for the given aperture of the 3.6-m DOT were calculated considering the transmission coefficients from the mirrors (M1 reflecting area $\sim 72 \%$, M1 reflectively $\sim 92 \%$ (350 850nm), M2 reflectively $\sim 86 \%$ (400 1000nm)), filters $(\sim 62 \%, 70 \%, 88 \%, 80 \%$, and $88 \%$ in Bessel $U, B, V, R$, and $I$ bands, respectively) and the CCD glass (transmission $\sim 98 \%$ ). The sky-brightness values for Devasthal were $\sim 22.1,22.4,21.3,20.5$ and $18.9 \mathrm{mag} /{ }^{\prime \prime 2}$ in the Bessel $U, B, V, R$, and $I$ bands, respectively, whereas the atmospheric extinction coefficients were $\sim 0.49$, $0.32,0.21,0.13$ and $0.08 \mathrm{mag}$ in Bessel $U, B, V, R$, and $I$, respectively (Kumar et al. 2000). The considered values of the quantum efficiencies for the STA CCD chip were $\sim 75 \%, 91 \%, 96 \%, 81 \%$ and $60 \%$ in the Bessel $U, B, V, R$, and $I$ bands, respectively.

Assuming that each optical photon will be able to produce a corresponding photo-electron and 


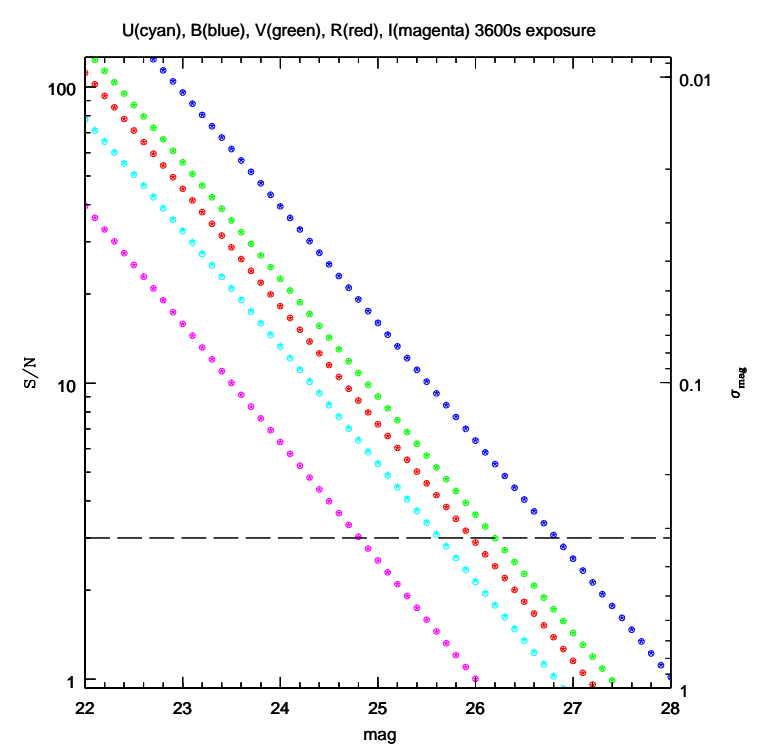

Figure 7: A simulated plot of the magnitude (X-axis) versus the signal-to-noise ratio (Y-axis, left) and corresponding error in the magnitude determinations (Y-axis, right) based on the throughput calculations (Mayya 1991) for the combination of the 3.6-m DOT and the $4 \mathrm{~K} \times 4 \mathrm{~K}$ CCD camera with a pixel size of $15 \mu \mathrm{m}$. The plot is reproduced for the set of Bessel $U B V R I$ filters and for an exposure time of 1 hour each. The value of the seeing is assumed to be $1.1^{\prime \prime}$ along with other standard values of parameters given in above table.

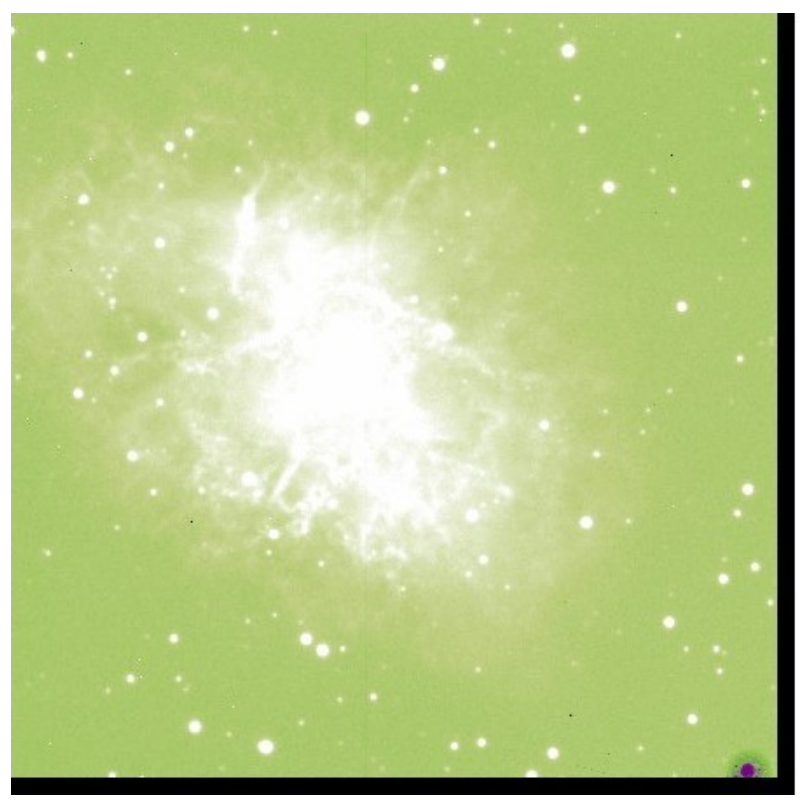

Figure 8: A $V$-band image of the Crab nebula (a supernova remnant) with an exposure time of $180 \mathrm{~s}$ taken using the $4 \mathrm{~K} \times 4 \mathrm{~K} \mathrm{CCD}$ camera mounted at the axial port of the 3.6-m DOT on 11 December 2015 during the testing phase. 


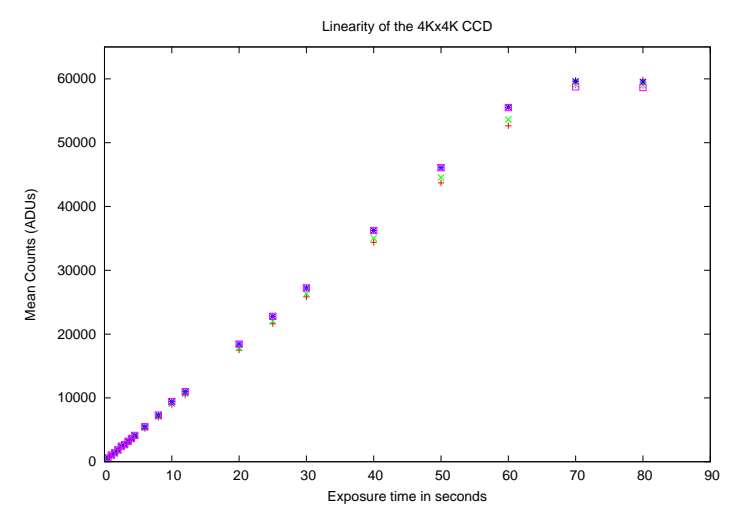

Figure 9: Linearity plot of the $4 \mathrm{~K} \times 4 \mathrm{~K} \mathrm{CCD}$ camera showing a linear behavior of the exposure time with counts up to $90 \%$ of the full-well. The four different symbols show the data taken at four different areas within the CCD frame taken at a speed of $1 \mathrm{MHz}$ and a gain value of $1 \mathrm{e}^{-} /$ADU.

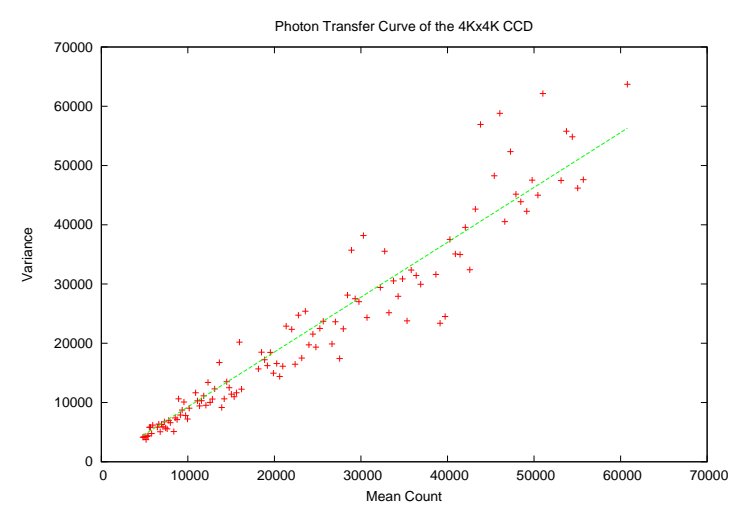

Figure 10: Photon transfer curve of the $4 \mathrm{~K} \times 4 \mathrm{~K}$ CCD Imager taken at a gain value of 1 and read out speed of $1 \mathrm{MHz}$. The measured value of the gain is $0.93 \pm 0.03 \mathrm{e}^{-} / \mathrm{ADU}$.

that the standard values of the CCD parameters like pixel size, dark current, read out noise, gain along with respective values given in above paragraph were used to calculate expected number of counts both from point sources and sky at zenith for an assumed value of seeing of $1.1^{\prime \prime}$, using the formula given in (Mayya 1991, McLean 1989). The signal-to-noise ratios were also calculated for stars of different brightness (Deep 2011). In Fig. 7, we have plotted such a result for a specific set of parameters (as described in above paragraph) for an assumed value of the exposure time of $3600 \mathrm{sec}$ for reference.

\section{CCD Characterization}

The $4 \mathrm{~K} \times 4 \mathrm{~K}$ CCD camera was characterized both in laboratory and in open sky conditions while mounted at the axial port of the 3.6-m DOT near the end of 2015. The Imager mounted at the axial port of the 3.6-m DOT on 11 December 2015 is shown in Fig. 8. For characterization purposes, several sky flat frames in different filters along with bias frames were taken for various combinations of the read-out speeds and gain values (they can be selected through the GUI of the camera control software). In general, over-scan areas were saved along with the frames to get additional information related to the possible fluctuations caused by the electronics. Based on the observed data, the bias stability was found as per specifications of the LN2 hold time. The standard deviations in observed flat frames were found to be $<2 \%$ in general. The linearity of the CCD was verified using the data obtained in November 2015. The results are shown in Fig. 9.

The photon transfer curve (PTC) was generated using the flat and bias frames taken at read out speed of $1 \mathrm{MHz}$ and gain setting of $1 \mathrm{e}^{-} / \mathrm{ADU}$ as shown in Fig. 10. The slope value between the mean counts and variance (calculated in a small and cleaned region of CCD frames) comes out to be $0.93 \pm 0.03$, pretty close to the gain value of $1 \mathrm{e}^{-} / \mathrm{ADU}$. Similar PTCs were also calculated for gain settings of 3 and $5 \mathrm{e}^{-} / \mathrm{ADU}$, giving rise to measured values of $2.82 \pm 0.08$ and $5.50 \pm 0.25$, respectively.

The gain and read out noise values were also verified occasionally using the two formulas as 
described in Howell (2006). In the formulas below, $\bar{B}$ is the mean value in an area of a given bias image, $\bar{F}$ is the mean value in an area of a given flat field, $\sigma_{\left(B_{1}-B_{2}\right)}$ is the standard deviation of pixels into the area of the difference of two bias images and $\sigma_{\left(F_{1}-F_{2}\right)}$ is the standard deviation of pixels into the area of the difference of the two flat frames.

$$
\begin{gathered}
\text { gain }=\frac{\left(\bar{F}_{1}+\bar{F}_{2}\right)-\left(\bar{B}_{1}+\bar{B}_{2}\right)}{\sigma_{\left(F_{1}-F_{2}\right)}^{2}-\sigma_{\left(B_{1}-B_{2}\right)}^{2}} \\
\text { readout noise }=\frac{\text { gain } \times \sigma_{\left(B_{1}-B_{2}\right)}}{\sqrt{2}}
\end{gathered}
$$

Using standard routines of IRAF, the derived values of the gain and readout noise values using the above two formulas were found to be consistent to those derived using the PTCs method. The first scientific image of the Crab nebula taken by the $4 \mathrm{~K} \times 4 \mathrm{~K} \mathrm{CCD}$ is given in Fig. 8 . More details about the characterization of the CCD camera will be published separately.

\section{Calibrations and First light results}

On 23 March 2017, images of globular cluster NGC 4147 and several Landolt standard fields were acquired both in open and closed loop conditions in various filters along with bias and flat frames. The range of exposure times were 30 to $600 \mathrm{~s}$ in various filters to cover both bright and faint point sources. Using IRAF/DAOPHOT standard routines, circularity and radial profiles were checked and pre-processing was done for both the NGC 4147 and Landolt PG standard fields. The FWHM of stars are nearly similar within the single frame of observations. The range of FWHM varies from frame to frame from $1.6^{\prime \prime}$ to $1.2^{\prime \prime}$ later in the night as the seeing improved.

We also attempted to calibrate the globular cluster field NGC 4147. We acquired data in Bessel $B$ and $R$ filters with an exposure time of $60 \mathrm{~s}$ to $600 \mathrm{~s}$ to cover both bright and faint stars in the field. For the calibration purposes, we also acquired the Landolt standard fields PG 1525 and PG 1528 in the Bessel $B V R$ filters having a good color range around similar airmass values.

The bias and flat frames observed in respective filters were used to pre-process the images using standard IRAF routine called "ccdproc", after having trimmed the images to remove the over-scan area. We used the "cosmicrays" routine to remove the cosmic hits in various exposures and images were stacked wherever required. After the pre-processing is done, we used the DAOPHOT-II FORTRAN subroutines in sequential standard orders (i.e. daogrow, ndaomatch, ndaomster, nccdstd, nccdlib, nfinal etc.) to calculate the transformation coefficients determined using the observed Landolt standard fields. These transformations were then applied to the observed globular cluster field NGC 4147 to calibrate the field. The calibrated color magnitude diagram (CMD) of NGC 4147 (Fig. 11) obtained using this procedure was plotted in Fig. 12. The results are described in the caption. Atmospheric extinction coefficients in all 10 filters were also estimated by observing Landolt standard fields within an airmass range of 1.1 to 3.5 and the values obtained using the standard procedure were determined. The derived values of the extinction coefficients using the data taken during 16-17 April 2017 are listed in the last column of Table 2.

\section{Acknowledgments}

Authors thankfully acknowledge contributions from Er. Vishal Shukla, Dr. Amitesh Omar, Dr. Brijesh Kumar and the full technical team of the DOT project. Timely support and encouragements from 


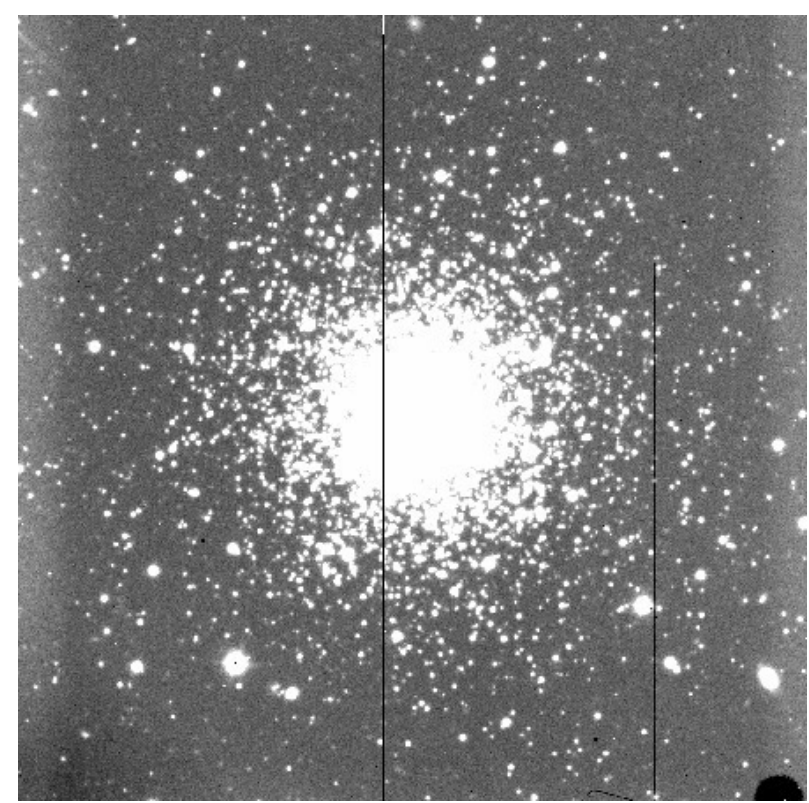

Figure 11: An example of the $B$-band frame pre-processed (cleaned, trimmed, and cosmicrays removed) of the globular cluster NGC 4147 taken in closed loop conditions having a single exposure of $600 \mathrm{~s}$ with the $4 \mathrm{~K} \times 4 \mathrm{~K}$ CCD Imager mounted at the axial port of the 3.6-m DOT on 23 March 2017.

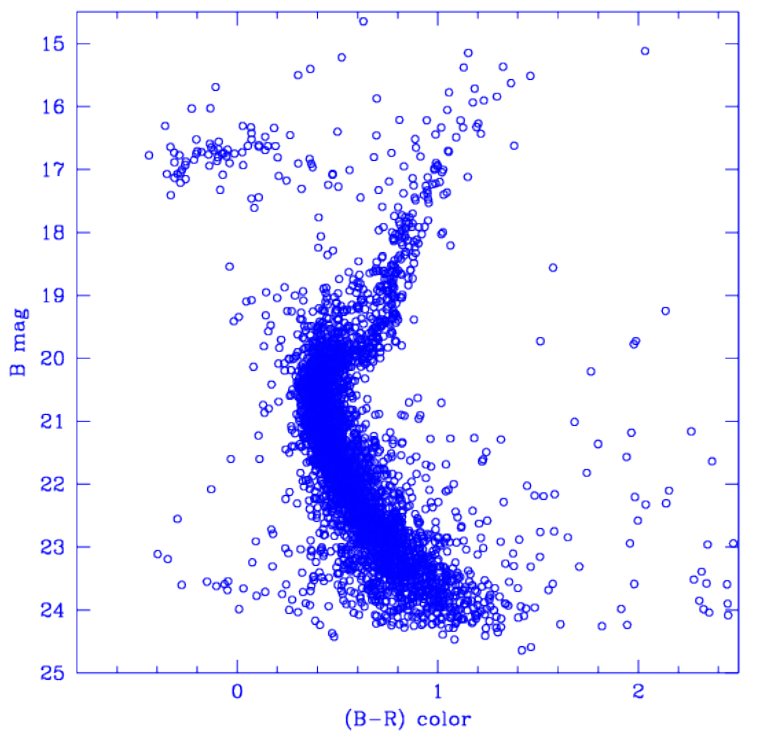

Figure 12: The $B$ versus $(B-R)$ colormagnitude diagram (CMD) of the Globular cluster NGC 4147 as obtained using the present calibration data taken using the $4 \mathrm{~K} \times 4 \mathrm{~K} \mathrm{CCD}$ mounted at the axial port of the 3.6-m DOT. The total number of common stars plotted (detected in both the filters) are around 3500 with a photometric accuracy of $<0.2 \mathrm{mag}$. In this figure, the number of detected stars having $B$ $<24$ mag are around 150 (with a photometric accuracy of $<0.2 \mathrm{mag}$ ) in an effective exposure time of $1200 \mathrm{~s}$. There are many more detections for which the error could not be established using the present data set. The main sequence branch and other features typical for this cluster are clearly identified using our data set observed in the Bessel $B$ and $R$ filters. 
Dr. A. K. Pandey, Dr. Wahab Uddin, Prof. Ram Sagar, PMB members and the Governing council are acknowledged for their consistent guidance throughout and to make this in-house instrument working for the astronomical community. It is suggested to cite this article while using data taken with the $4 \mathrm{~K} \times 4 \mathrm{~K}$ CCD Imager.

\section{References}

Alonso-Garcia J., Mateo M., Sen B. et al. 2012, AJ, 143, 70

Bekki K., Forbes D. A. 2006, A\&A, 445, 485

Cameron P. B., Chandra P., Ray A. et al. 2004, Nature, 434, 1112

Deep A., Fiorentino G., Tolstoy E. et al. 2011, A\&A, 531, 151

Friel E. D. 1995, ARA\&A, 33, 381

Fabian A. C. 2012, ARA\&A, 50, 455

Howell S. B. 2006, Handbook of CCD Astronomy, second edition, ISBN 13978-0-521-85215-9

Joshi S., Mary D. L., Martinez P. et al. 2006, A\&A, 455, 303

Joshi Y. C., Pandey A. K., Narasimha D., Sagar R. 2005, A\&A, 433, 787

Kalirai J. S., Strader J., Anderson J. et al. 2008, ApJ, 682, 37

Kann D. A., Klose S., Zhang B. et al. 2011, ApJ, 734, 96

Kroupa P. 2002, Science, 295, 82

Kumar P., Zhang B. 2015, Physics Reports, 561, 1109

Kumar B., Sagar R., Rautela B. S. et al., Bull. Astr. Soc. India, 28, 675

Mayya Y. D. 1991, Journal of Astrophysics and Astronomy 12, 319

McLean I. S. 1989, in Electronic and Computer-Aided Astronomy, Ellis Horwood Chichester

Pandey A. K., Mahra H. S. 1986, Ap\&SS, 120, 107

Pandey A. K., Durgapal, A. K., Bhatt B. C. et al. 1997, A\&AS, 122, 111

Pandey A. K., Sharma S., Ogura K. et al., 2008, MNRAS, 383, 1241

Pandey J. C., Singh K. P., Drake S. A. et al. 2005, Astronomical Journal, 130, 1231

Pandey S. B. 2006, PhD Thesis

Pandey S. B. 2013, JApA, 34, 157

Pandey S. B., Sahu, D. K., Resmi, L. et al. 2003, Bull. Astr. Soc. India, 31, 19

Pant P, Stalin C. S., Sagar R. 1999, A\&AS, 136, 19

Pasquini L., Belloni T. 1998, A\&A, 336, 902

Sagar, R., Kumar B., Omar A., Pandey A. K. 2012, SPIE, 8444E, 1TS

Sagar R., Pandey S. B. 2012, Astronomical Society of India Conference Series, 5, 1

Sagar, R., Subramaniam A., Richtler T. et al. 1999, A\&A Suppliment, 135, 391

Sagar R., Piskunov A. E., Miakutin V. I., Joshi U. C. 1986, MNRAS, 220, 383

Samuel J. G., Ian R. S. 2011, Bull. Astr. Soc. India, 39, 539

Saurabh S., Pandey A. K., Pandey J. C. et al. 2012, PASJ, 64, 107

Singal A. K., Konar C., Saikia D. J. 2004, MNRAS, 347, L79

Sreekumar P. 2005, Bull. Astr. Soc. India, 33, 253

Stalin C. S., Sagar R., Pant P. et al. 2001, Bull. Astr. Soc. India, 29, 39

Stalin C. S., Gopal-Krishna, Sagar R., Wiita P. J. 2004, MNRAS, 350, 175

Swarup G., Ananthakrishnan S., Kapahi V. K. et al. 1991, Current Science, 60, 2

Harris W. E., Racine R. 1979, ARA\&A, 17, 241

Yadav R. K. S., Kumar B., Subramaniam A. et al. 2008, MNRAS, 390, 985 